\title{
Neutrophil gelatinase-associated lipocalcin (NGAL) as a biomarker of dialysis-dependent acute kidney injury following infrarenal aortic surgery
}

Helene Korvenius Jørgensen*, Jannie Bisgaard and Torben Gilsaa

*Correspondence: helene_j@yahoo.com

Lillebaelt Hospital Kolding, Department of Anaesthesiology and Intensive Care, Denmark.

\begin{abstract}
Background: Acute kidney injury (AKI) is common following abdominal aortic surgery. NGAL might be useful in the early diagnosis of AKI since it responds rapidly to ischaemic damage.

Methods: Twenty patients undergoing elective infrarenal aortic surgery. U-NGAL was measured before surgery and 24,48 and 72 hours postoperatively.

$\underline{\text { Results: }}$ No significant rise in U-NGAL was seen in patients without AKI at any time. All patients who developed dialysisdependent AKI had a significant increase in U-NGAL.

Conclusion: U-NGAL did not increase solely in response to the surgical trauma. U-NGAL may be a useful biomarker for AKI following abdominal aortic surgery.

Keywords: Acute kidney injury, abdominal aortic surgery, intensive care
\end{abstract}

\section{Introduction}

Abdominal aortic surgery (AAS) is a high-risk procedure with a relatively high incidence of postoperative acute kidney injury (AKI). The diagnosis of AKI has traditionally been based on an increase in se-creatinine. However, se-creatinine may not increase until up to $50 \%$ of the kidney function is lost [1-4] making timely interventions difficult. Animal studies have shown that AKI caused by ischaemic injury can be prevented and treated, but it is crucial that therapies are instituted early [4]. NGAL increases rapidly as a response to ischaemic injuries in the kidneys [1-4], but NGAL is also a marker of inflammatory activation [1]. The ischaemia-reperfusion injury and subsequent inflammatory response to aortic cross clamping may by itself induce a rise in NGAL, in spite of preserved renal function. If so, NGAL would be an unreliable marker of AKI following AAS.

The aim of this study is to describe the changes in NGAL after AAS in patients with and without postoperative dialysisdependent AKI.

\section{Methods}

Approval from the local ethics committee was obtained. Twenty patients undergoing open elective infrarenal AAS using aortic cross clamping were included consecutively after written informed consent. NGAL was measured in urine samples before surgery (T0), and 24 (T24), 48 (T48) and 72 hours (T72) postoperatively. Urine samples were centrifuged at $2000 \mathrm{~g}, 20^{\circ}$ $\mathrm{C}$ for 10 minutes and supernatant frozen at $-80^{\circ} \mathrm{C}$. U-NGAL was quantified using an immunoassay (BioPorto, Gentofte, Denmark).
The upper normal limit for this analysis is given as $250 \mathrm{ng} / \mathrm{mL}$ by the manufacturer. Median NGAL values were compared using Mann-Whitney u-test, and for repeated measurements Friedman test was used.

\section{Results}

No significant rise in U-NGAL was seen in patients without AKI at any time. Three patients developed dialysis-dependent AKI. Two of these patients required acute dialysis from day 1 and 3 respectively, and one died from multi organ failure on day 3 . In these patients U-NGAL increased significantly $(p<0,05)$. In patients without AKI, U-NGAL remained below the threshold value at all times and no significant increase was found over time $(p=0.08)$. This indicates that the operation per se had no influence on U-NGAL ( Figure 1 and Table 1).

\section{Discussion}

The aim of this study was to investigate whether the surgical trauma caused by aortic cross clamping could in itself cause an increase in U-NGAL, thus making it a less useful marker of AKI in this setting. The results found in this study show that U-NGAL levels did not increase above threshold value solely as a response to surgery. Only patients who developed dialysisdependent AKI showed a significant increase in U-NGAL.

There are certain limitations to this study. Due to the small sample sizes this study is primarily observational. Only three patients developed dialysis-dependent AKI and NGAL-values seen in these patients had a wide variation. This variation in 
Jørgensen et al. Journal of Anesthesiology and Clinical Science 2013, http://www.hoajonline.com/journals/pdf/2049-9752-2-16.pdf

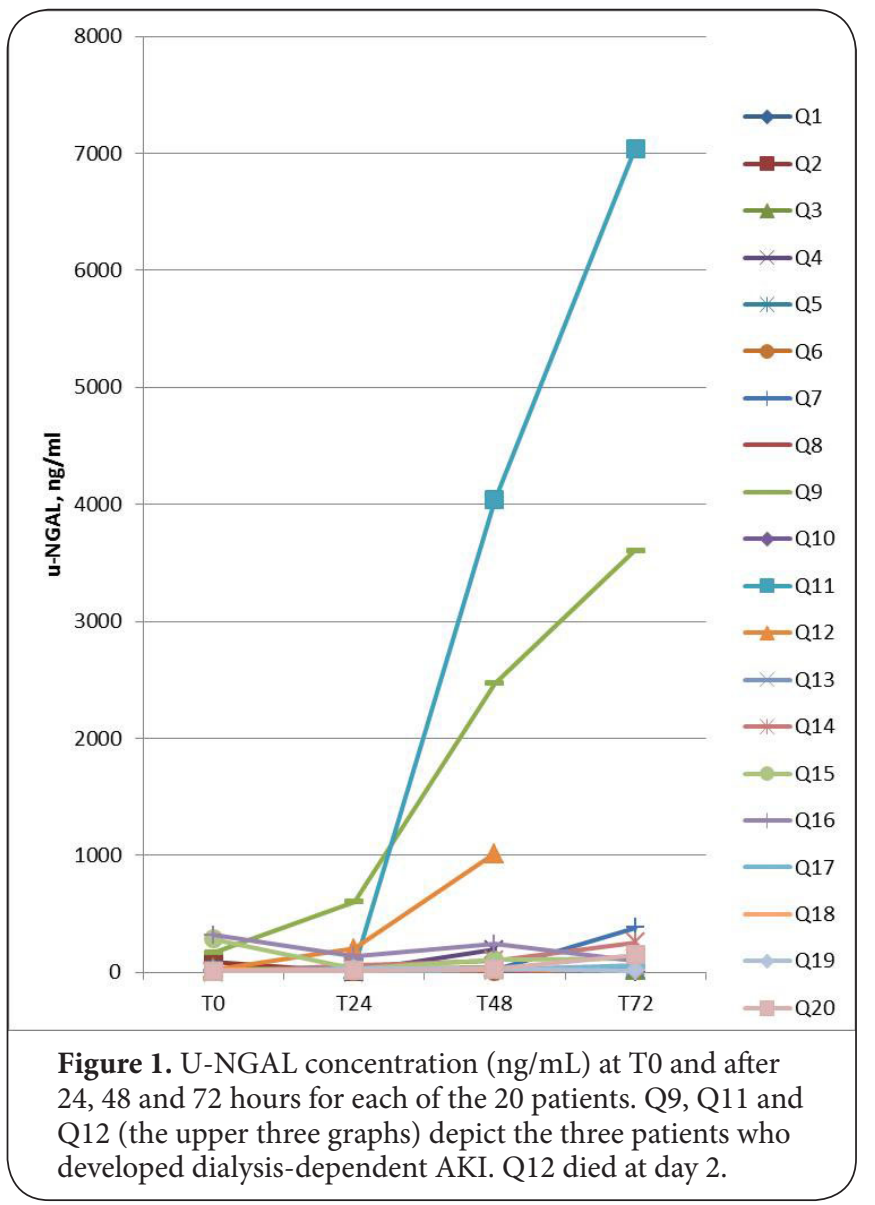

Table 1. U-NGAL values (ng/mL) for patients with and without dialysis dependent AKI. Results are shown as median(inter-quartile range).

\begin{tabular}{lll}
\hline & No dialysis, $\mathbf{n}=\mathbf{1 7}$ & Dialysis, $\mathbf{n}=\mathbf{3}$ \\
\hline T0 & $10(6-22)$ & $18(16-168)$ \\
T24 & $17(6-31)$ & $203(47-603)$ \\
T48 & $25(18-73)$ & $2473(1017-4036)$ \\
T72 & $50(28-117)$ & $5323(3606-7039)$ \\
\hline
\end{tabular}

NGAL-values could be caused by a number of factors: varying degrees of kidney injury between the patients, variation in comorbidity or by the fact that initiation of dialysis is a subjective decision made by the attending doctor. This study was not designed to clarify this, but further research into the course of U-NGAL in patients who develop dialysis-dependent AKI is recommended.

In this study we only looked at AKI severe enough to require dialysis. Using the RIFLE criteria based on increase in p-creatinine and decreases in urinary output, it is likely that more patients could have been diagnosed with milder degrees of AKI despite normal U-NGAL.

Diagnostics based on urinary samples have advantages, namely being non-invasive and containing few interfering proteins [4], but U-NGAL is useless in patients with anuria. Studies evaluating se-NGAL in this patient group would be of value.

Future studies should include measurements of U-NGAL at earlier time points, for example every second hour after aortic clamping to describe the early course of NGAL and to determine its potential as a very early biomarker. Mishra and colleagues [4] showed, in their study of a paediatric cardiac surgery population, that U-NGAL obtained two hours postsurgery was the most powerful predictor of postoperative AKI.

Based on the present findings, it can be concluded that U-NGAL did not increase solely as a response to the surgical trauma. U-NGAL may thus be a useful biomarker for the early diagnosis of acute kidney injury following abdominal aortic surgery.

\section{Competing interests}

The authors declare that they have no competing interests.

\section{Publication history}

Received: 16-Dec-2012 Revised: 17-Jan-13

Accepted: 24-Feb-2013 Published: 02-Mar-2013

\section{Reference}

1. Kim T, Arnaoutakis GJ, Bihorac A, Martin TD, Hess PJ, Jr., Klodell CT, Tribble CG, Ejaz AA, Moldawer LL and Beaver TM: Early blood biomarkers predict organ injury and resource utilization following complex cardiac surgery. J Surg Res 2011, 168:168-72. | Article | PubMed

2. Parikh CR, Jani A, Mishra J, Ma Q, Kelly C, Barasch J, Edelstein CL and Devarajan P: Urine NGAL and IL-18 are predictive biomarkers for delayed graft function following kidney transplantation. Am J Transplant 2006, 6:1639-45. | Article I PubMed

3. Lebkowska U, Malyszko J, Lebkowska A, Koc-Zorawska E, Lebkowski W, Malyszko JS, Kowalewski R and Gacko M: Neutrophil gelatinaseassociated lipocalin and cystatin $\mathrm{C}$ could predict renal outcome in patients undergoing kidney allograft transplantation: a prospective study. Transplant Proc 2009, 41:154-7. I Article I PubMed

4. Mishra J, Dent C, Tarabishi R, Mitsnefes MM, Ma Q, Kelly C, Ruff SM, Zahedi K, Shao M, Bean J, Mori K, Barasch J and Devarajan P: Neutrophil gelatinase-associated lipocalin (NGAL) as a biomarker for acute renal injury after cardiac surgery. Lancet 2005, 365:1231-8. | Article | PubMed

\section{Citation:}

Jørgensen H K, Bisgaard J and Gilsaa T: Neutrophil gelatinase-associated lipocalcin (NGAL) as a biomarker of dialysis-dependent acute kidney injury following infrarenal aortic surgery. journal of Anesthesiology and Clinical Science 2013, 2:16. http://dx.doi.org/10.7243/2049-9752-2-16 